\title{
Linear Response of Hamiltonian Chaotic Systems as a Function of the Number of Degrees of Freedom
}

\author{
Marco Bianucci and Riccardo Mannella \\ Dipartimento di Fisica dell'Università di Pisa and Istituto Nazionale Fisica della Materia, UdR Pisa Piazza Torricelli 2 , \\ 56100 Pisa, Italy \\ Paolo Grigolini \\ Dipartimento di Fisica dell'Università di Pisa and Istituto Nazionale Fisica della Materia, UdR Pisa Piazza Torricelli 2 , 56100 \\ Pisa, Italy, Department of Physics, University of North Texas, P.O. Box 5368, Denton, Texas 76203, and Istituto di Biofisica del \\ Consiglio Nazionale delle Ricerche, Via San Lorenzo 28, 56127 Pisa, Italy
}

(Received 26 January 1996; revised manuscript received 8 May 1996)

\begin{abstract}
Using numerical simulations we show that the response to weak perturbations of a variable of Hamiltonian chaotic systems depends on the number of degrees of freedom: When this is small $(\approx 2)$ the response is not linear, in agreement with the well known objections to the Kubo linear response theory, while, for a larger number of degrees of freedom, the response becomes linear. This is due to the fact that increasing the number of degrees of freedom the shape of the distribution function, projected onto the subspace of the variable of interest, becomes fairly "regular." [S0031-9007(96)00795-8]
\end{abstract}

PACS numbers: 05.45.+b, 05.20.-y, 05.70.-a

It is well known that linear response theory (LRT) is a key ingredient in the theoretical approach to the microscopic foundation of nonequilibrium and equilibrium statistical mechanics and thermodynamics [1]. For linear response we mean that the average value of a variable of interest $B$ can be expressed as a linear function of the external disturbances: If we assume that in the absence of external perturbations the distribution function of the system is the equilibrium one (canonical, microcanonical, etc., depending on the case considered) and that the average value of $B$ with respect to this distribution is zero, then after the time $t=0$ at which the perturbation of intensity $K$ is switched on, the distribution will no longer be in equilibrium and the average of $B$ will change. In general we can write

$$
\langle B\rangle_{K}(t)=K \chi_{B}(t)+O\left(K^{2}\right),
$$

where $\langle\cdots\rangle$ means an average with respect to the appropriate distribution (herein, the microcanonical one), the subscript $K$ reminds us of the presence of the perturbation (of intensity $K$ ) and $\chi_{B}(t)$ is the susceptibility for the macroscopic variable $\langle B\rangle$. Ohm's law or the various linear relations between polarization and electromagnetic fields are well known examples of linear response behavior. The LRT is an attempt to derive these linear relations from a microscopic and Hamiltonian treatment of the system under study. A microscopic derivation of the susceptibility $\chi(t)$ in Eq. (1) is then obviously equivalent to a microscopic foundation of the LRT. The most famous LRT is that of Kubo [2]. Kubo used a perturbative treatment of the Liouville equation

$$
\frac{\partial}{\partial t} \rho(t)=\left(\mathcal{L}_{0}+K \mathcal{L}_{I}\right) \rho(t),
$$

where $\rho(t)$ is the distribution function of the system, $\mathcal{L}_{0}$ the unperturbed Liouvillian (for Hamiltonian systems the Liouvillian operator is given by the Poisson brackets with the Hamiltonian $\left.H: \mathcal{L} \cdot=\{H, \cdot\}_{\mathrm{PB}}\right)$, and $\mathcal{L}_{I}$ the perturbation. The approach of Kubo is appealing since it leads to expressions for the susceptibility in terms of time integral of correlation functions:

$$
\chi_{B}^{\mathrm{Kubo}}(t)=\int_{0}^{t}\left\langle B_{0}(\tau) \mathcal{L}_{I}\right\rangle_{0} d \tau
$$

where

$$
B_{0}(\tau) \equiv e^{\mathcal{L}_{0}^{+} \tau} B
$$

is the unperturbed evolution of $B$. The Kubo LRT leads to elegant theoretical predictions, which have been found to date to be always in remarkable agreement with experiments and numerical simulations [3-5]. The LRT theoretical predictions have been confirmed in experiments on the shear viscosity of Lennard-Jones fluids $[6,7]$ and on dielectric behavior of polymer systems involving the measurement of both equilibrium and dynamic properties [8]. The numerical experiments of response to a weak field have led to the accurate calculation of transport coefficients like the electrical conductivity $[9,10]$. In a recent Letter, Chernov et al. [11] provided the first derivation of Ohm's law based on a deterministic mechanical model (the Lorentz model) and proved the validity of the Kubo LRT in that context.

The clear success of the Kubo LRT contrasts with the well known criticism of van Kampen [12], based on the fact that the Liouville and Hamilton descriptions seem to be equivalent. This would imply that Kubo's approach is essentially equivalent to deriving the linear macroscopic properties from the linearization of the classical trajectories and, due to the highly unstable character of the microscopic trajectories, this linearization is possible only in the limiting case of perturbations of virtually vanishing intensity. The aim of this Letter is to clarify the role that the number of degrees of freedom of 
nonlinear Hamiltonian systems plays to make the linear response possible.

Our Hamiltonian chaotic systems are characterized by a positive Lyapunov exponent $\lambda$, which sets a typical time scale $\bar{t} \equiv 1 / \lambda$. We set $B=x-\langle x\rangle_{0}$ where $x$ is a given space variable of our system, and we use as a perturbation an external potential $K x$, defining the intensity of the perturbation by the parameter $K$. We introduce $\epsilon$, the energy per degree of freedom, and denote by $\omega$ a characteristic frequency of the system. Rephrasing van Kampen's argument [12] for our case, in order to have linear response of the individual trajectories at a time $t$ after the application of the perturbation, we have the condition

$$
K\left(e^{\lambda t}-1\right) \ll \omega \sqrt{\epsilon} .
$$

This means that to have linear response at macroscopic times $t, K$ must be extremely small, and that the use of "macroscopic" $K$ 's confines the linear response regime to the microscopic time range, i.e., for $t \sim \bar{t}$.

On the other hand, it was shown $[13,14]$ that no quantitative difference between "macroscopic" and "microscopic" linearity is possible: The system responds linearly on a macroscopic time scale as long as the Kubo LRT applies, and if the system responds linearly then it must do so obeying the Kubo LRT. It was also shown with very simple geometrical arguments [13] that mixing Hamiltonian systems with a finite volume in phase space responds linearly to external fields of macroscopic intensities, not only for microscopic times less than $\bar{t}$, but also for very large times. The argument is that the distribution function for mixing Hamiltonian systems with a finite volume in phase space evolves toward a constant function on the hypersurface of given energy $E$ (microcanonical distribution). Using this property we have that for large times the average value of $B$ depends only on the geometry of this hypersurface and that its shape, defined by $\delta(E-H)=0$, is a smooth function of the Hamiltonian parameters: Thus $\langle B\rangle$, for $t \rightarrow \infty$, is a well behaved function of $K$ and, in particular, it is linear in $K$ for small (but still macroscopic) $K$ 's.
The susceptibility given by Eq. (3) is then either correct at all times or at most there is a discrepancy between Eq. (3) and the real response only in the region of intermediate times, where the system does not respond linearly at all. This latter statement was checked in detail elsewhere [13]. Here we will show that in case of a "small" dynamical system and in the presence of a breakdown at intermediate times, there is the hope of recovering a linear response also in the region of discrepancy, thus making the Kubo LRT true for all times if the number of degrees of freedom is conveniently increased. In other words, it would be the size of the physical system the fundamental ingredient which makes the LRT valid or invalid.

We study the linear response to the "external" perturbation $K x$ of the following $\beta$ Fermi-Pasta-Ulam ( $\beta$-FPU) [15-21] systems

$$
H_{0}=\sum_{i=1}^{N} \frac{w_{i}^{2}}{2}+W\left(y_{N}\right)+\sum_{i=2}^{N} W\left(y_{i}-y_{i-1}\right),
$$

where

$$
W(y) \equiv \frac{y^{4}}{4}+\frac{y^{2}}{2}
$$

and $w_{i} \equiv \dot{y}_{i}, i=1, \ldots, N, y_{1} \equiv x, w_{1} \equiv v$. The systems studied have $N=2,4,8$, respectively. We set the energy $\epsilon$ per oscillator $\epsilon=10$ for $N=8$ and $N=4$, and $\epsilon=20$ for $N=2$. According to Refs. [18-20] this should be a convenient choice to produce a regime of fully developed chaos: The calculation of the largest Lyapunov exponent for the different cases yields $\lambda=0.15,0.11$, and 0.11 , respectively. The unperturbed dynamics of the system corresponding to these energy densities, within the accuracy of a numerical treatment, is mixing and hence ergodic. Thus for fixed total energy $E \equiv N \epsilon$ we have that the equilibrium distribution of these systems is the microcanonical distribution. Using Eq. (3) (with $\mathcal{L}_{I}=\partial / \partial v$ ), we obtain the following Kubo-like expression for the susceptibility [13]:

$$
\int d \mathbf{y} d \mathbf{w} \tilde{x} \int_{0}^{t} e^{\mathcal{L}_{0} \tau} \frac{\partial}{\partial v} \frac{\delta\left(E-H_{0}\right)}{A_{0}(E)} d \tau \chi(t)=\left(\frac{\partial \ln A_{0}(E)}{\partial E}\right)\left(\langle\tilde{x} \tilde{x}(t)\rangle_{0}-\left\langle\tilde{x}^{2}\right\rangle_{0}\right)+\frac{\partial}{\partial E}\left(\langle\tilde{x} \tilde{x}(t)\rangle_{0}-\left\langle\tilde{x}^{2}\right\rangle_{0}\right),
$$

with $\tilde{x} \equiv x-\langle x\rangle_{0}$ and

$$
A_{0}(E) \equiv \int \delta\left(E-H_{0}\right) d \mathbf{y} d \mathbf{w}
$$

It may be argued that the variable chosen here to study the response $(x)$ is not macroscopic, and that we should use something like $\bar{x} \equiv \sum_{1}^{n} y_{i}$ instead. However, it is clear from the structure of Eq. (8) that this choice would simply lead to a sum of terms similar to those in Eq. (8).
Notice that the result for the susceptibility in Eq. (8) obtained using the microcanonical distribution is different from the one derived by Kubo [2] based on the canonical distribution. In the standard Kubo LRT (i.e., in the canonical case) the decay of the susceptibility towards the equilibrium values is guided by the autocorrelation function of the variable $x$, whereas here it also involves a term proportional to the derivative with respect to the total energy of the autocorrelation function. Given that 
the total energy is an extensive quantity, it is expected $[1,13]$ that when $N$ is large this additional term vanishes (approximately like $1 / N$ ) and Eq. (8) becomes identical to the standard Kubo result [2].

We integrated our $\beta$-FPU systems using a fourth order symplectic Runge-Kutta algorithm [22]. The integration time step was chosen so that the total energy was always conserved with at least eight significant figures throughout the simulations. For all systems studied we used an external field with intensities for degrees of freedom in the range $0.1 \leq K / N \leq 0.2$, i.e., macroscopic values much larger than the microscopic $K$ 's allowed by the van Kampen argument [see Eq. (5)]; these $K$ values are small enough to guarantee, using geometric arguments, linear response for large times [13]. Note that we used a $K$ proportional to $N$ to prevent the "washing out" of the external field intensity due to the increase in the number of degrees of freedom. The choice of the perturbation $K x$ is due to numerical convenience, but our arguments based on the phase-space structure would not be affected by a different choice, like the perturbation $K \sum_{1}^{N} y_{i}$, implying the coupling to a macroscopic variable. As we can see in Fig. 1 the response $\langle\tilde{x}\rangle_{K} / K$ for $N=2$ is linear only for small and large times, while it is not so for intermediate times, in agreement with the results of [13]. It is then striking that simply increasing the number of degrees of freedom from two to four, we have that the system responds linearly even in the intermediate times region (see Fig. 2). Then the response remains linear at any time when the number of degrees of freedom is further increased (Fig. 3). We expect the same property to be shared by all the systems as chaotic as that here studied. From Fig. 4 it is clear that even for the largest value of $N$ used (i.e., $N=8$ ), the number of degrees of freedom is

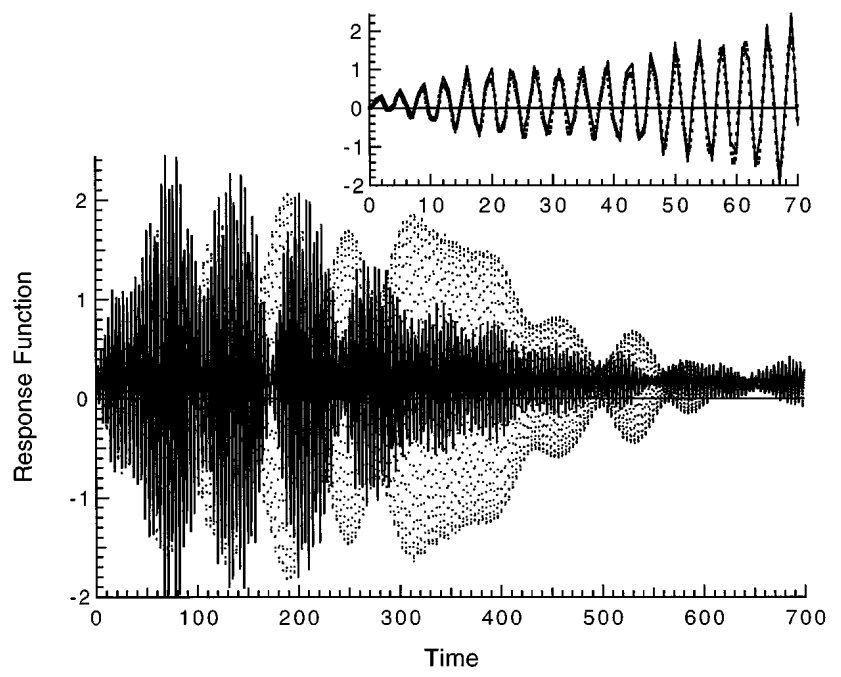

FIG. 1. Time dependent susceptibility (solid line) for a $\beta$ FPU system with $N=2$, compared to the theoretical predictions (dots). The total energy of the chain is taken to be $E=40$. Inset: A blowup of the short times region. not large enough to disregard the derivative with respect to the total energy of the autocorrelation function of the variable of interest [see Eq. (8)], i.e., the LRT is the one corresponding to microcanonical rather than canonical systems. Thus $N=4,8$ are numbers large enough for the system to respond linearly to external perturbations but not yet enough to use the canonical distribution as an approximation of the microcanonical distribution.

The intuitive reason why an increase in the number of degrees of freedom should linearize the response over the whole time scale even for systems where the microscopic dynamics is highly chaotic is clear: The chaoticity of the dynamics shows up in the distribution through the development of whorls and tendrils, which quickly lead to a fragmentation of the distribution. These fine elements are responsible for the nonlinear response to the external perturbation. At large times this fragmentation is very pronounced, to the point that the whorls or tendrils are so small and so uniformly distributed that in the evaluation of averages of the variable of interest the distribution is indistinguishable from a uniform distribution. This implies, though, that this final distribution is no longer unstable towards the external perturbation, and hence that the variable of interest responds linearly. At intermediate times the coarse graining of the distribution function can still be macroscopic, leading in principle to a macroscopic nonlinear response; however, what matters in the evaluation of the response is the projection of the distribution function onto the subspace of the variable of interest: This projection is equivalent to an average, where many details of the (complex) structure of the equilibrium distribution are lost. The projection is obviously smoother the larger the number of degrees taken, leading to a response more and more linear. Our numerical simulations, carried out for Hamiltonian systems with an extremely chaotic and nonlinear microscopic dynamics, lend credit to this conjecture. It is also clear that, very interestingly, the linear

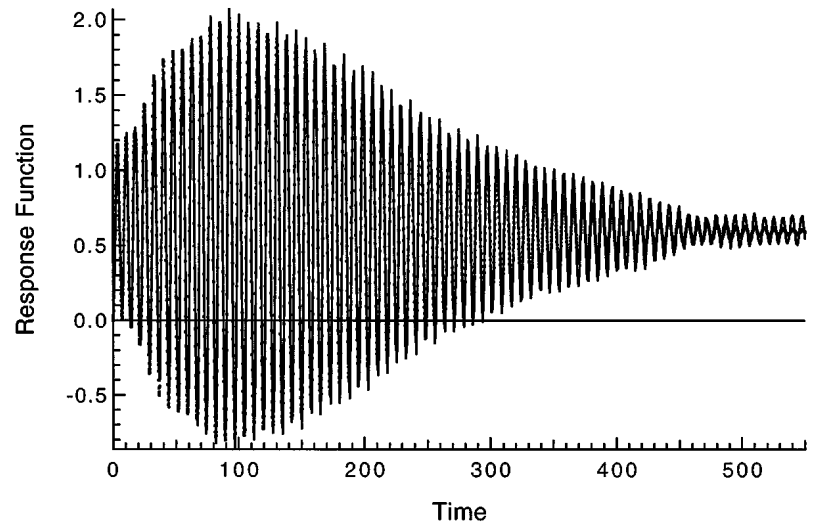

FIG. 2. Time dependent susceptibility (solid line) for a $\beta$ FPU system with $N=4$, compared to the theoretical predictions (dots, barely visible under the solid line). The total energy of the chain is taken to be $E=40$. Clearly the system responds linearly at all times. 


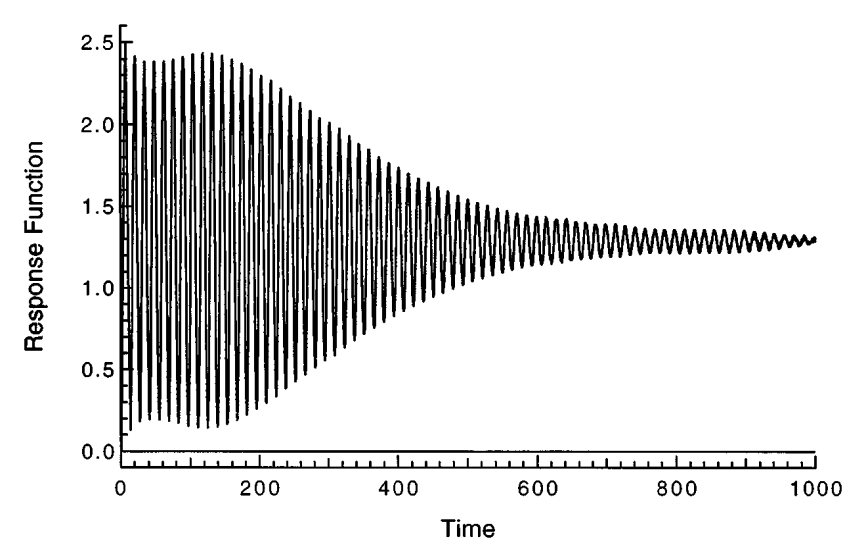

FIG. 3. Time dependent susceptibility (solid line) for a $\beta$ FPU system with $N=8$, compared to the theoretical predictions (dots). The total energy of the chain is taken to be $E=80$. See comments to Fig. 2 .

response of the system is recovered well before the "standard" form of LRT (canonical as opposite to microcanonical) is applicable.

In conclusion, it is arguable that in a generic macroscopic system (for which $N \gg 2$ ) there are so many degrees of freedom that the linear response to external perturbations should be the norm rather than the exception, even if the microscopic dynamical system is strongly nonlinear. Care must be exerted in analyzing data because the response may differ substantially from the standard one given by Kubo LRT, even in a linear response regime. It is arguable that a breakdown of LRT should perhaps be more usefully interpreted as an insight on the smallness

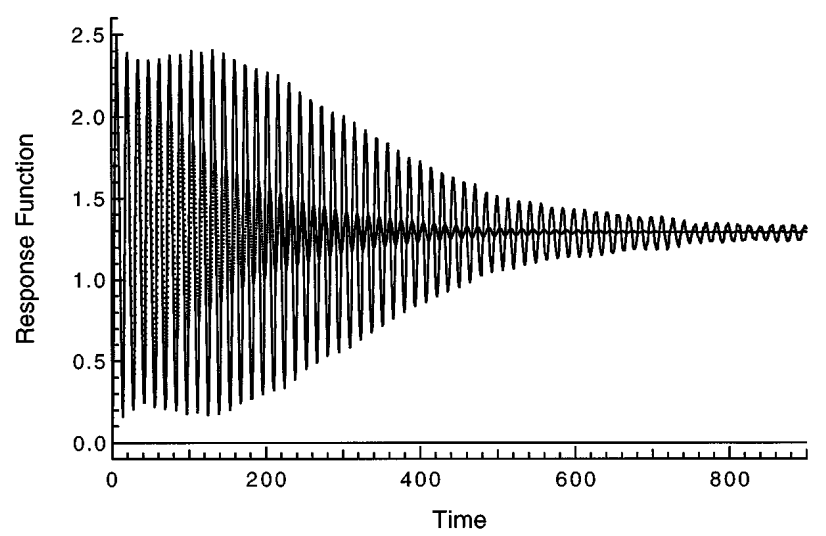

FIG. 4. Comparison between the theoretical susceptibility (solid line) for a $\beta$-FPU system with $N=8$, obtained using Eq. (8), and the theoretical susceptibility obtained assuming that the system is described by a canonical statistics (dots). The total energy of the chain is taken to be $E=80$. It is clear that the system, although linear at all times (see Fig. 3), is not described by a canonical statistics. of the degrees of freedom involved in the response rather than on the strong nonlinearity of microscopic dynamics.

[1] M. Bianucci, R. Mannella. B. J. West, and P. Grigolini, Phys. Rev. E 51, 3002 (1995).

[2] R. Kubo, M. Toda, and N. Hashitsume, Statistical Physics (Springer, Berlin, 1985), Vol. 2, p. 198.

[3] D. J. Schneider and J.H. Freed, Adv. Chem. Phys. LXXIII, 387 (1989).

[4] Simulation of Liquids and Solids, edited by G. Ciccotti, D. Frenkel, and I. R. McDonald (North-Holland, Amsterdam, 1987).

[5] Memory Function Approaches to Stochastic Problems in Condensed Matter, edited by M. W. Evans, P. Grigolini, and G.P. Parravicini, Advances in Chemical Physics Vol. LXII (Wiley, New York, 1985), p. 65.

[6] E. M. Gosling, I. R. McDonald, and K. Singer, Mol. Phys. 26, 1475 (1973).

[7] W. T. Ashurst and W. G. Hoover, Phys. Rev. Lett. 31, 206 (1973).

[8] M. Cook, D. C. Watts, and G. Williams, Trans. Faraday Soc. 66, 2503 (1970).

[9] G. Ciccotti and G. Jacucci, Phys. Rev. Lett. 35, 789 (1975).

[10] G. Ciccotti, G. Jacucci, and I. R. McDonald, Phys. Rev A 13, 426 (1976).

[11] N. I. Chernov, G. L. Eyink, J.L. Lebowitz, and Ya.G. Sinai, Phys. Rev. Lett. 70, 2209 (1993).

[12] N. G. van Kampen, Phys. Nor. 5, 279 (1971).

[13] M. Bianucci, R. Mannella, B. J. West, and P. Grigolini, Phys. Rev. E 50, 2630 (1994).

[14] M. Falcioni and A. Vulpiani, Physica (Amsterdam) 215A, 481 (1995).

[15] E. Fermi, J. Pasta, and S. Ulam, Los Alamos Report No. LA-1940, 1955, later published in Collected Papers of Enrico Fermi, edited by E. Segré (University of Chicago Press, Chicago, 1965), Vol. II, p. 978; also reprinted in Nonlinear Wave Motion, edited by A. C. Newell, Lectures in Applied Mathematics Vol. 15 (American Mathematical Society, Providence, Rhode Island, 1974); also in The Many-body Problem, edited by D.C. Mattis (World Scientific, Singapore, 1993).

[16] F. M. Izrailev and B. V. Chirikov, Dokl. Acad. Nauk SSSR 166, 57 (1966) [Sov. Phys. Dokl. 11, 30 (1966)].

[17] B. V. Chirikov, F. M. Izrailev, and V. A. Tayursky, Comput. Phys. Commun. 5, 11 (1973).

[18] M. Pettini and M. Landolfi, Phys. Rev. A 41, 768 (1990).

[19] M. Pettini and M. Cerruti-Sola, Phys. Rev. A 44, 975 (1991).

[20] L. Casetti and M. Pettini, Phys. Rev. E 48, 4320 (1993).

[21] R. Livi, A. Politi, and S. Ruffo, J. Phys. A 19, 2033 (1986).

[22] H. Yoshida, Phys. Lett. A 150, 262 (1990). 